\title{
Emerging applications of stereotactic radiotherapy in head and neck cancer
}

\author{
Minh Tam Truong, M.D., ${ }^{1}$ Gregory Grillone, M.D.,${ }^{2}$ Christine Tschoe, M.D.,$^{3}$ \\ Lawrence Chin, M.D., ${ }^{3}$ Lisa Ann Kachnic, M.D., ${ }^{1}$ and Scharukh Jalisi, M.D. ${ }^{2,3}$ \\ Departments of ${ }^{1}$ Radiation Oncology, ${ }^{2}$ Otolaryngology, and ${ }^{3}$ Neurosurgery, Boston Medical Center, \\ Boston University School of Medicine, Boston, Massachusetts
}

\begin{abstract}
Advances in the management of locally advanced head and neck cancer (HNC) have been focused on treatment intensification, including concomitant chemoradiotherapy, biological agents, and combining surgery with chemoradiotherapy. Despite these improvements, locoregional recurrence still constitutes the main pattern of treatment failure. As improvements in radiotherapy delivery and image-guided therapy have come to fruition, the principles of stereotactic radiosurgery are now being applied to extracranial sites, leading to stereotactic body radiotherapy. This article focuses on the emerging evidence for the use of stereotactic body radiotherapy for treatment of $\mathrm{HNC}$ as a boost after conventional external-beam radiotherapy, and also as reirradiation in recurrent or second primary HNC. (DOI: 10.3171/2009.9.FOCUS09199)
\end{abstract}

\section{KeY Words - head and neck cancer - stereotactic body radiotherapy - stereotactic radiosurgery • reirradiation • dose escalation}

$\mathrm{A}$ CCORDING to the American Cancer Society, 35,720 new cases and 7600 deaths from HNC are estimated to occur in the US in $2009 .{ }^{5}$ Head and neck cancers comprise approximately $5 \%$ of all cancers ${ }^{36}$ and ranks among the lowest patient survival rates of the major cancer groups. The therapeutic management of HNC is challenging due to the many critical normal structures that control functions such as vision, speech, swallowing, taste, and cosmesis, all of which can be affected by locally advanced disease presentation and treatment selection. The 5-year survival rate for locally advanced HNC ranges from 30 to $75 \% \%^{4,7,14,20,23}$ depending on the disease type, location, stage, and extent, and patient characteristics. Standard treatment for locally advanced tumors is often multimodal involving combinations of surgery, radiotherapy, and chemotherapy. Local control is the primary goal of treatment, as local recurrence occurs in

\footnotetext{
Abbreviations used in this paper: $\mathrm{EBRT}=$ external-beam radiation therapy; EORTC = European Organisation for Research and Treatment of Cancer; FDG-PET = fluorodeoxyglucose-PET; GTV = gross tumor volume; $\mathrm{HNC}=$ head and neck cancer; LINAC = linear accelerator; PTV = planning target volume; $\mathrm{QOL}=$ quality of life; SBRT = stereotactic body radiotherapy; SRS = stereotactic radiosurgery.
}

$30-50 \%{ }^{23,37}$ and carries a very poor prognosis. However, maintaining QOL and functional outcome is also of paramount importance.

In the past decade, improvements in HNC local control, survival, and functional organ preservation have been achieved with altered radiotherapy fractionation regimens (e.g., concomitant boost or hyperfractionation), ${ }^{26}$ concomitant chemoradiation strategies for sites including the nasopharynx, oropharynx, larynx, and hypopharynx, ${ }^{2-4,23,25,43}$ as well as with increased radiation sensitization utilizing biological agents ${ }^{10,11}$ such as cetuximab, a monoclonal antibody against the epidermal growth factor receptor. Advances in surgical techniques have also led to surgical organ preservation strategies. ${ }^{41,60}$ Improvements in radiotherapy delivery via conformal techniques, such as intensity-modulated radiation therapy, ${ }^{15}$ have improved the therapeutic ratio by reducing dose to normal tissue ${ }^{58}$ while improving delivery of radiation to the target tumor. However, complications of therapy such as xerostomia, dysphagia, and osteoradionecrosis can still adversely affect the QOL of long-term survivors. Despite these technological advances, approximately $30-83 \%$ of these patients still die due to persistent disease, locoregional recurrence, or second primary tumor, ${ }^{21,37,39,57}$ hence local control of HNC continues to be a therapeutic challenge. 


\section{T. Truong et al.}

\section{Rationale for Radiotherapy Dose Escalation in HNC}

The optimal radiation dose to effectively control $\mathrm{HNC}$ has been limited by the normal tissue tolerance of adjacent organs, rather than reaching the tumoricidal dose required for complete sterilization of the cancer. Methods to improve the therapeutic ratio include dose escalation by SRS or brachytherapy (catheters surgically placed into the tumor and loaded with radiation sources). Integration of a planned radiation boost following conventional EBRT has been used in base of tongue and oral tongue cancers using brachytherapy. Studies using a brachytherapy boost have demonstrated superior local control compared with EBRT alone; cumulative doses up to 80 Gy provide 5 -year local control rates of approximately $80 \% .6,9,28,32,38$ However, with the introduction of conformal EBRT delivery techniques, including intensity-modulated radiation therapy and its associated improved normal tissue sparing, brachytherapy has been used less often for a variety of reasons such as patient inconvenience, the complexity and dependence on the individual physician's surgical technique and experience, its invasiveness, the potential increased cost, hospitalization requirements, and radiation exposure to personnel.

Stereotactic radiosurgery has been used for skullbase tumors and also in nasopharyngeal cancer ${ }^{18}$ using the same principles of intracranial radiosurgery, using the skull as the main reference for immobilization of the target. Stereotactic radiosurgery traditionally required invasive immobilization by means of a stereotactic head frame to ensure complete immobilization of the patient and thereby permitting focused high-dose radiation beam delivery to the target lesion in 1 fraction. In nasopharyngeal cancer, an SRS boost following a conventional course of chemoradiation therapy with EBRT using a LINAC gantry-based SRS boost was demonstrated in a patient series at Stanford University; ${ }^{42,58} 45$ patients with nasopharyngeal cancer were treated to a median dose of 12 Gy in 1 fraction (range 7-15 Gy). All patients experienced local tumor control at a follow-up of 31 months. Late complications included 3 incidences of cranial nerve zoster, 3 patients with transient V2 or V3 numbness (12-24 months after SRS), 1 patient reporting paresis in the third cranial nerve, 1 reported radiation-related retinopathy, and asymptomatic temporal lobe necrosis was found in 3 patients who had intracranial disease extension. In their subsequent follow-up series, 33 patients were treated with frame-based LINAC gantry-based SBRT and 49 patients were treated with a CyberKnife (Accuray) nasopharyngeal boost with excellent local control rates..$^{30}$ Overall, the 5-year rate for freedom from local relapse was $98 \%$. Late toxicity was acceptable with radiation retinopathy in 3 patients, carotid aneurysm in 1 patient, and radiographic temporal lobe necrosis in 10 patients ( 9 of whom had T-4 tumors).

In a single-institution report by Yau et al., ${ }^{63}$ a fractionated stereotactic boost using a median dose of $15 \mathrm{~Gy}$ at 6-8 Gy per fraction delivered 2-3 times per week was more effective at local control compared with a 20 Gy brachytherapy treatment for patients with persistent local disease. Ahn et al. ${ }^{3}$ used fractionated stereotactic radia- tion (the XKnife-3 system [Radionics] with a relocatable Gill-Thomas-Cosman frame) in 24 patients as a boost to conventional EBRT, and in 24 patients as the sole modality of treatment. Patients were treated using conventional fraction sizes of $2 \mathrm{~Gy}$ per fraction to a total dose of $80 \mathrm{~Gy}$. The 2-year local control and survival rates were 92 and $60 \%$, respectively.

Stereotactic radiosurgery may also be considered for salvage treatment of recurrent nasopharyngeal cancers, ${ }^{17,49,58,62}$ with outcome results comparable to patients undergoing brachytherapy and surgery. Chua et al. ${ }^{17}$ reported a series of 18 patients treated for persistent local or recurrent nasopharyngeal cancer, using LINAC-based SRS. A median dose of 12.5 Gy (range 11-14 Gy) in 1 fraction, with a median target volume of $5.3 \mathrm{~cm}^{3}$ (range $2.2-16.9 \mathrm{~cm}^{3}$ ), was used. An $89 \%$ complete response rate was achieved, confirmed by nasoendoscopy and biopsy, of which only 4 patients experienced a subsequent relapse. One patient was noted to have temporal lobe necrosis, although this was attributed to the initial course of EBRT rather than the SRS. Xiao et al.$^{62}$ reported a series of 50 patients treated for recurrent or residual nasopharyngeal cancer with fractionated stereotactic radiation, with a complete response in $76 \%$ using varying fractionation schedules, to a median dose of 24 Gy (range 14-35 Gy). In a case series of LINAC gantry-based radiosurgery for locally recurrent nasopharyngeal cancer, ${ }^{13} 3$ patients were treated after a full course of radiation therapy with single-fraction SRS. While 1 patient was disease-free after treatment, 2 patients developed serious neurological sequelae for which it was uncertain whether this represented a treatment-related complication.

Thus, single-fraction SRS in patients with nasopharyngeal cancer who have already received conventional fractionated doses up to a total dose of 70 Gy may carry significant risk of long-term sequelae. Whereas LINAC gantry-based SRS boost using a non-relocatable head frame appears to be associated with promising response rates, its application is limited only to the nasopharyngeal site due to its proximity to the skull base and also due to limitations in immobilization requirements. Table 1 summarizes studies examining SRS boost in nasopharyngeal cancer.

\section{Salvage Options for Recurrent HNC}

Locally recurrent primary HNCs that have already received a full course of EBRT present a specific therapeutic challenge due to limited management options. In most cases, the normal surrounding tissues have approached close to accepted radiotherapy tolerance doses. Any additional EBRT dose required to achieve sterilization of persistent tumor carries a significant risk of treatment-related morbidities including spinal cord injury, cranial nerve injury, osteoradionecrosis requiring surgery, fistula, and vascular injury such as carotid artery hemorrhage. When feasible, salvage surgery is the treatment of choice, with studies indicating a 5-year survival rate of $35 \%$ in early locally recurrent tumors ${ }^{61}$ and $16 \%$ in advanced locally recurrent disease. ${ }^{29}$ When salvage surgery is not feasible, chemotherapy alone with or without biological therapy, and conventional EBRT reirradiation with or without 
Emerging applications of SBRT in head and neck cancer

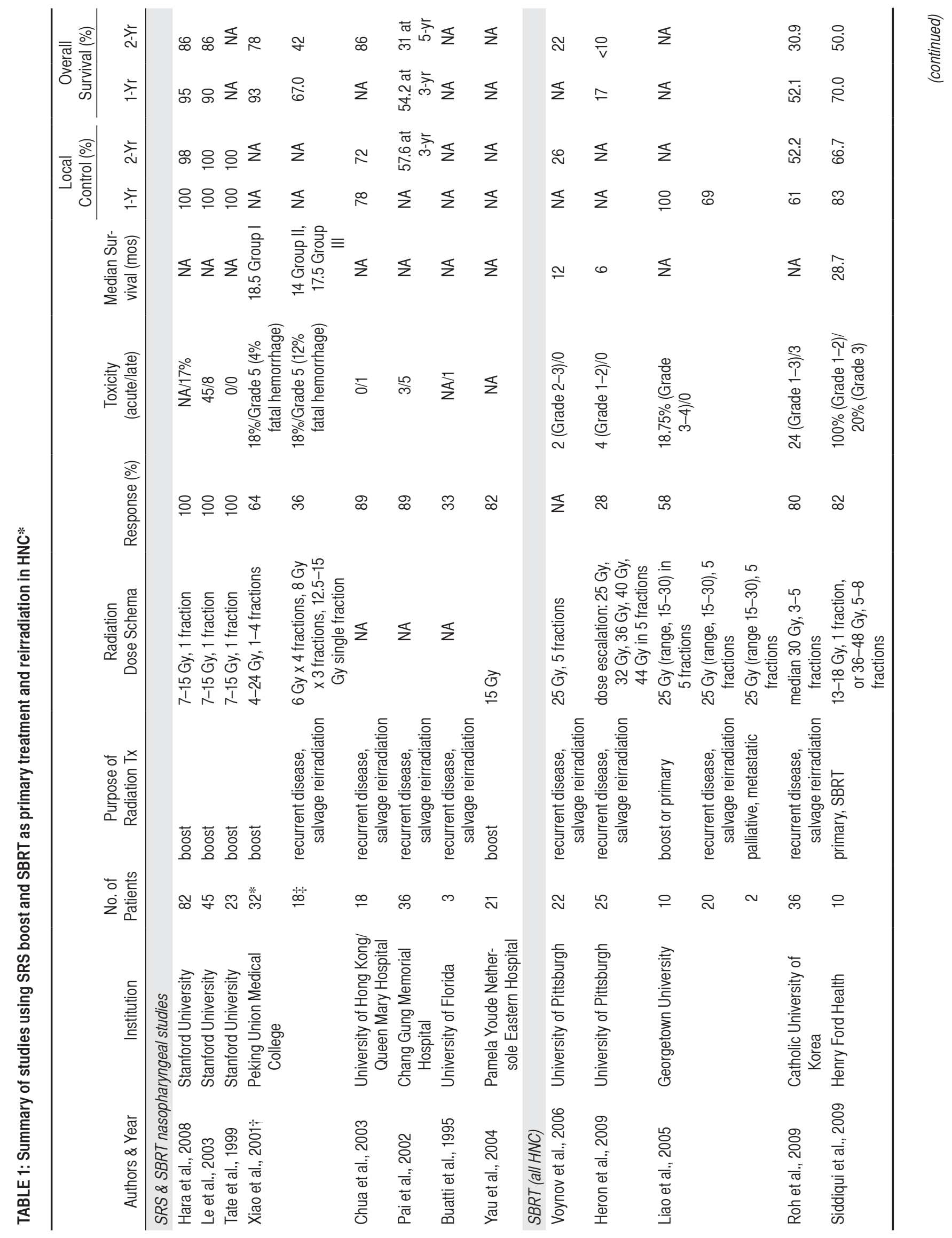




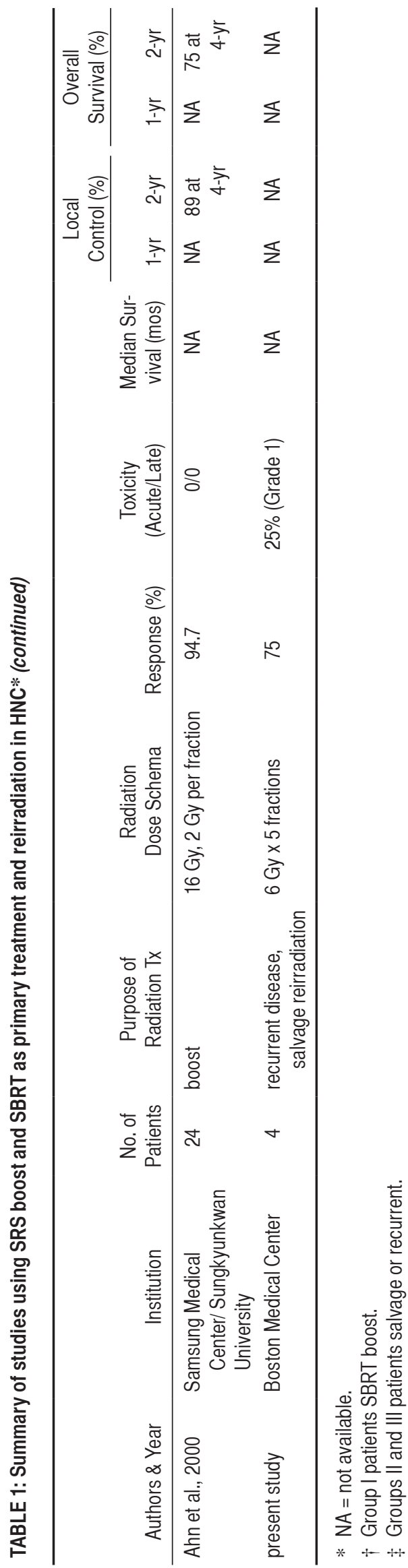

chemotherapy, are the only other options. Phase I and Phase II investigations of salvage reirradiation and concurrent chemotherapy approaches report median survival rates of only 8-10 months and 2-year survival rates of 15$35 \%$. $^{16,22,31,39,50,53-55}$ In 1 patient series, an $11 \%$ treatment mortality rate was noted. ${ }^{31}$ Therefore, while conventional EBRT using 3D conformal reirradiation approaches may be acceptable for properly selected patients with limited salvage therapy options, they are associated with substantial acute and late morbidities. ${ }^{22,44,50,57}$

\section{Applications of SBRT in HNC}

Linear accelerator gantry-based and Gamma Knife intracranial SRS use invasive head frames, which can often be uncomfortable and poorly tolerated. To date, LINAC and Gamma Knife SRS have limited application for extracranial locations including $\mathrm{HNC}$, largely due to limitations in immobilization requirements and difficulty in tracking movement of potentially mobile organs, making it difficult to apply the concept of rigid immobilization to nonrigid structures. Non-skull-based HNC sites are subject to movement with talking, swallowing, and breathing, and therefore make it difficult to target without adjusting for all aspects of organ motion. As methods to track tumor and patient motion during treatment and advances in physics and radiation delivery have come to fruition, application of the principles of SRS that have been used for many decades to treat intracranial and skull-based conditions can now be applied extracranially to HNC. This application has given rise to SBRT, which uses certain anatomic references (such as the skull, spine, or surgically placed fiducials) as a stereotactic localizer to guide highly focused radiation beams to the tumor in an extracranial site, while maintaining maximal normal tissue sparing to minimize toxicity. Because an invasive stereotactic head frame is no longer required, SBRT can be fractionated, and fractionation potentially allows larger volume targets to be treated compared to SRS.

Stereotactic body radiotherapy in HNC can be delivered by LINAC gantry-based systems using relocatable, noninvasive, stereotactic frame localization ${ }^{52}$ and also robotic radiosurgery systems. Robotic radiosurgery systems such as the CyberKnife mount a lightweight 6-MV LINAC on a robotic arm capable of motion with 6 degrees of freedom. ${ }^{2,40}$ Frameless radiosurgery systems rely on real-time image-guided systems to ensure accurate localization and compensate for patient external motion and internal tumor motion throughout treatment delivery, and deliver high precision SRS or SBRT without the use of an invasive non-relocatable stereotactic frame. ${ }^{2}$

\section{Emerging Evidence for SBRT in Recurrent HNC}

Because conventional methods of reirradiation have been limited by significant toxicity, attempts to find methods to minimize normal tissue irradiation have led to exploration of SBRT in HNC. Voynov et al..$^{59}$ demonstrated the feasibility of CyberKnife SBRT in 22 patients diagnosed with recurrent HNC, who were treated between 2001 and 2004. The 2-year local control rate was $26 \%$, 
with a median patient survival of 12 months. These investigators reported only Grade 2-3 mucositis and no acute Grade 4-5 toxicities. No late toxicities occurred. In a follow-up patient series from the same institution, Heron et al. ${ }^{34}$ studied the safety and efficacy of SBRT in patients with squamous cell HNC in a Phase I dose escalation trial of 25 patients. Five dose tiers were tested using the CyberKnife, starting from 25 Gy up to a maximum dose of $44 \mathrm{~Gy}$, in 5 fractions over 2 weeks. Treatment sites included the nasopharynx (1 patient), oropharynx (6 patients), larynx (10 patients), oral cavity (7 patients), and other (1 patient). Median tumor volume was 44.8 $\mathrm{cm}^{3}$. With a follow-up of 13 months, toxicity was limited to Grade 1-2. The overall response rate was $28 \%$ with a maximum duration of response of 4 months; 12 of 25 patients had stable disease and 4 patients had progressive disease. The response rate was found to be independent of dose and volume. Patterns of treatment failure were either in field failures, or completely outside of the field. The investigators did not note any marginal failures, suggesting this method of SBRT is accurate in terms of beam delivery, although the optimal dose of radiation required to sterilize the tumor may be higher than their tested maximum dose.

Roh et al. ${ }^{51}$ used CyberKnife SBRT to treated 36 patients (in 44 sites) with recurrent, unresectable, previously irradiated HNC, using a median dose of 30 Gy (range $18-40$ Gy). Fractionation schema varied from 30 Gy in 3 fractions to $35-40$ Gy in 5 fractions. Median tumor size was $22.6 \mathrm{~cm}^{3}$ (range $0.2-114.9 \mathrm{~cm}^{3}$ ). Treatment sites included the nasopharynx (8 patients), maxillary sinus (8 patients), cervical lymph nodes ( 8 patients), skull base ( 7 patients), nasal cavity (4 patients), retropharyngeal lymph nodes (3 patients), orbit ( 2 patients), tongue (2 patients), oropharynx (1 patient), and parotid gland (1 patient). With a median follow-up of 17.3 months, 15 patients achieved complete tumor regression, and 13 showed partial tumor regression. Local recurrence-free survival at 1 and 2 years was 61 and $52.2 \%$, respectively. The 1- and 2-year overall survival rate was 52.1 and $30.9 \%$, respectively. Twentyfour of the 35 patients developed acute complications, including Grades I-III mucositis, nausea, or subacute otitis media, and Grade II dermatitis. Long-term adverse events were observed in 3 patients, including trismus, soft-tissue necrosis, skull-base bone necrosis, and mandibular bone necrosis. One patient died of skull-base necrosis, which was believed to be a treatment-related late complication. This patient received 33 Gy in 3 consecutive daily fractions. As a result, the authors recommended that a lower dose per fraction and increasing fractionation from 3 to 5 fractions should be used.

In a patient series from Georgetown University, 32 patients were treated with CyberKnife SBRT at 37 sites with primary and recurrent HNC. ${ }^{45}$ The median total dose was 25 Gy (range 15-30 Gy) prescribed to the $75 \%$ isodose line, in 5 fractions. The median cumulative radiation dose to the treated site (including prior courses of radiation) was $84 \mathrm{~Gy}$ (range $28-158 \mathrm{~Gy}$ ). A local control rate of $81 \%$ was achieved. Grades 3 and 4 toxicity was observed in 6 patients. The median tumor volume treated was $52.9 \mathrm{~cm}^{3}$ (range $4.1-723.2 \mathrm{~cm}^{3}$ ).
Stereotactic body radiotherapy employing a LINAC gantry-based system (BrainLAB, Inc.) was studied in a series of 44 patients with 55 lesions, with primary recurrent $\mathrm{HNC}$ and metastatic disease to the head and neck region. ${ }^{52}$ Fractionated SBRT was used in 37 patients and the remainder received single-dose SBRT. Indications for using SBRT as the primary treatment in this study included comorbidities that precluded patients from undergoing surgery, conventional radiation, or refusal of another form of treatment. These investigators found a 1- and 2-year local control rate in the primary treatment group of 83.3 and $66.7 \%$, respectively. For recurrent $\mathrm{HNC}$, the corresponding 1- and 2-year local control rate was 60.6 and $40.4 \%$, respectively. The median treatment volume was $15.5 \mathrm{~cm}^{3}$ (range 1.8-155 $\mathrm{cm}^{3}$ ). Dose fractionation schema varied, from single doses of 14,16 , and $18 \mathrm{~Gy}$, to fractionated doses of 36 Gy in 6 fractions, to 48 Gy in 8 fractions 2-3 times weekly. Radiation toxicity included Grade 1-2 mucositis in all patients with oropharyngeal or laryngeal lesions. Grades 3 and 4 toxicity was observed in 7 patients, including facial pain in 1 patient, cataract formation in 1 patient, dysphagia in 2 patients, fistula in 2 patients, and mandibular necrosis in 1 patient.

These studies demonstrate that there is a range of dose fractionation schemes feasible in treating a variety of different HNC sites with SBRT as a form of reirradiation. Based on these collective data, 5 fraction regimens of doses between 30 and 44 Gy appear to have the best toxicity profile, although further studies are needed to validate these findings. The literature regarding SBRT for HNC is summarized in Table 1.

\section{Initial Boston Medical Center Experience Using Robotic SBRT for Recurrent HNC}

At Boston Medical Center, 4 patients were treated between 2008 and 2009 in an open Phase I protocol examining reirradiation in $\mathrm{HNC}$. Eligible patients had either recurrent $\mathrm{HNC}$ or a second $\mathrm{HNC}$ (after treatment with a full course of EBRT for HNC) within the radiotherapy field. Diagnostic workup included a full medical history and physical examination, including examination under anesthesia using laryngoscopy, esophagoscopy, and bronchoscopy, as well as multidisciplinary evaluation by a head and neck surgeon and radiation oncologist. Histological confirmation of cancer was obtained in all patients. Metastatic workup included radiological evaluation of disease extent with an FDG-PET/CT scan. Median age at diagnosis of recurrent HNC was 63.5 years of age (range 54-75 years). There were 3 men and 1 woman. Prior HNC diagnoses included nasopharyngeal cancer (1 patient), tonsil cancer ( 1 patient), larynx cancer (1 patient), and base of tongue cancer (1 patient). Median time between prior radiotherapy and diagnosis of recurrent or second HNC was 12.4 years (range 1.9-30.1 years).

\section{Patient Selection}

Patients eligible for reirradiation using CyberKnife SBRT had localized disease recurrence or new primary HNC, without evidence of metastatic disease, and were not considered a surgical candidate or refused surgery. 
Treated sites included the base of the tongue (in 2 patients) and posterior hypopharyngeal wall (in 1 patient), and 1 patient had 2 localized primary cancers involving the tonsil and oral tongue, extending to the base of the tongue.

Other factors to consider for appropriate patient selection for reirradiation using SBRT in HNC included performance status, prior radiotherapy dose received to normal tissues, tumor size and site, proximity to critical normal structures (such as the spinal cord, larynx, carotid arteries, cranial nerves, brainstem, optic structures, mandible, and parotid gland), the presence of localized disease, and potential functional sequelae (such as speech and swallowing function) after treatment.

Stereotactic body radiotherapy used as a stereotactic boost has limited data for its routine use $\mathrm{e}^{13,17,49,58}$ and should generally be considered in patients who have persistent local disease after conventional EBRT in whom surgical options are limited.

\section{Defining the Target Volume}

Accurate target volume definition is of paramount importance for SBRT. Often imaging using CT alone may not accurately define the tumor and its margins. Positron emission tomography fused with CT improves the visualization of the tumor extent. ${ }^{12,33-35,51}$ Additional information from MR imaging can also improve target volume definition, ${ }^{27,48}$ especially in patients with nasopharyngeal cancer and skull-based tumors. ${ }^{24}$ After defining the GTV, a clinical target volume may be defined to account for potential subclinical disease and a PTV margin to account for treatment setup. In most patient series, ${ }^{34,52}$ no additional margins were created to account for setup error, because the SBRT systems used were within submillimeter accuracy. In the patient series studied by Roh et al., ${ }^{51}$ a margin of 2-3 $\mathrm{mm}$ beyond the FDG PET avid tumor was used to account for subclinical disease or potential internal organ motion.

Patients treated at Boston Medical Center on the reirradiation protocol using CyberKnife SBRT used ${ }^{18} \mathrm{~F}$ FDG-PET/CT scanning for all patients in the treatment position (immobilized in a custom thermoplastic mask). Patients then underwent radiation planning (simulation) with high-resolution CT. The GTV was defined by FDGPET/CT in all cases; additional imaging using MR imaging was used in 1 case to assist target delineation. A 5-mm expansion on the GTV was performed to create a PTV.

\section{Dosimetric Considerations}

After defining the target volumes including the GTV and PTV, critical normal tissues were contoured. Avoidance structures included optic structures, spinal cord, brainstem, carotid arteries, parotid glands, uninvolved larynx, and esophagus. For dose constraints in fractionated SBRT for HNC using 5 fractions, we used the same guidelines as conventional fractionated EBRT. Dose per fraction to the optic chiasm, optic nerves, spinal cord, and brainstem are kept below 2 Gy per fraction and prior dose received from EBRT should be cumulative. Cumulative dose constraints to the spinal cord should not exceed a total maximum point dose of $50 \mathrm{~Gy}$, optic chiasm tolerance of 50-54 Gy, and brainstem tolerance of $54 \mathrm{~Gy}$, with a maximum surface dose of $60 \mathrm{~Gy}$. If parotid gland sparing was not used with prior EBRT and the patient has preexisting xerostomia, then the parotid gland is not spared with SBRT. For carotid arteries we attempt to keep the dose below 3 Gy per fraction for a 5-fraction regimen. The larynx and epiglottis are kept below 4 Gy per fraction. Other considerations include the location of the target, prior EBRT dose, and time to recurrence. Very few published guidelines exist for hypofractionated SBRT for HNC; Heron et al. ${ }^{34}$ describe their dose constraints as follows: spinal cord $\leq 8 \mathrm{~Gy}$, larynx $\leq 20 \mathrm{~Gy}$, mandible $\leq 20$ Gy, parotid gland variable, brainstem $\leq 8 \mathrm{~Gy}$, oral cavity variable. The radiotherapy plan was devised using proprietary Accuray inverse planning software. Each plan consisted of noncoplanar static beams from a library of more than 1000 possible directions. Treatment consisted of 100-200 beams, producing a high radiotherapy dose to the target, but a steep falloff to surrounding healthy tissue. CyberKnife SBRT obviates invasive immobilization, although thermoplastic immobilization is used to minimize gross movements and excessive corrections needed by the robotic arm. Patients were treated to a total dose of 30 Gy in 5 fractions over 10 days using 6 Gy per fraction, usually prescribed to the $80 \%$ isodose line to encompass $>90-95 \%$ of the PTV. Figure 1 shows an isodose plan in axial, sagittal, and coronal planes in a patient with HNC.

\section{Tracking Target Motion During SBRT Delivery}

CyberKnife System. Robotic SBRT using the CyberKnife system uses radiological anatomic landmarks as the stereotactic reference point that can be seen on realtime continuous orthogonal radiographs. ${ }^{1}$ To account for movement, the CyberKnife must be able to track and compensate for any external or internal motion of the target. This can be done by 1 of 3 methods depending on the location of the tumor: fiducial, spine, or skull tracking. Tumor localization using fiducial markers to track the tumor requires placement of gold seed fiducials approximately 5 days prior to radiotherapy planning. Placement of the seeds and any edema caused by placement are allowed to resolve prior to acquisition of the planning $\mathrm{CT}$.

There are no studies to date examining the optimal placement of fiducials for HNC SBRT. At Boston Medical Center we continue to refine a protocol for appropriate fiducial placement for HNC SBRT. The challenges of fiducial placement include obtaining adequate exposure of the oral cavity and oropharynx, and the limited dimensions of the head and neck to allow multiple fiducial placement. The issues we consider include: 1) fiducials cannot be in the same plane when viewed by the CyberKnife image guidance radiographic units; 2) obtaining adequate exposure of the oropharynx for accurate fiducial placement; 3 ) at least 4 fiducials should be placed to allow for placement error; 4) the distance between each seed placement needs to be at least $1-\mathrm{cm}$ apart to allow them to be observed as individual reference points by the CyberKnife image guidance radiographic units; and 5) placement should be 


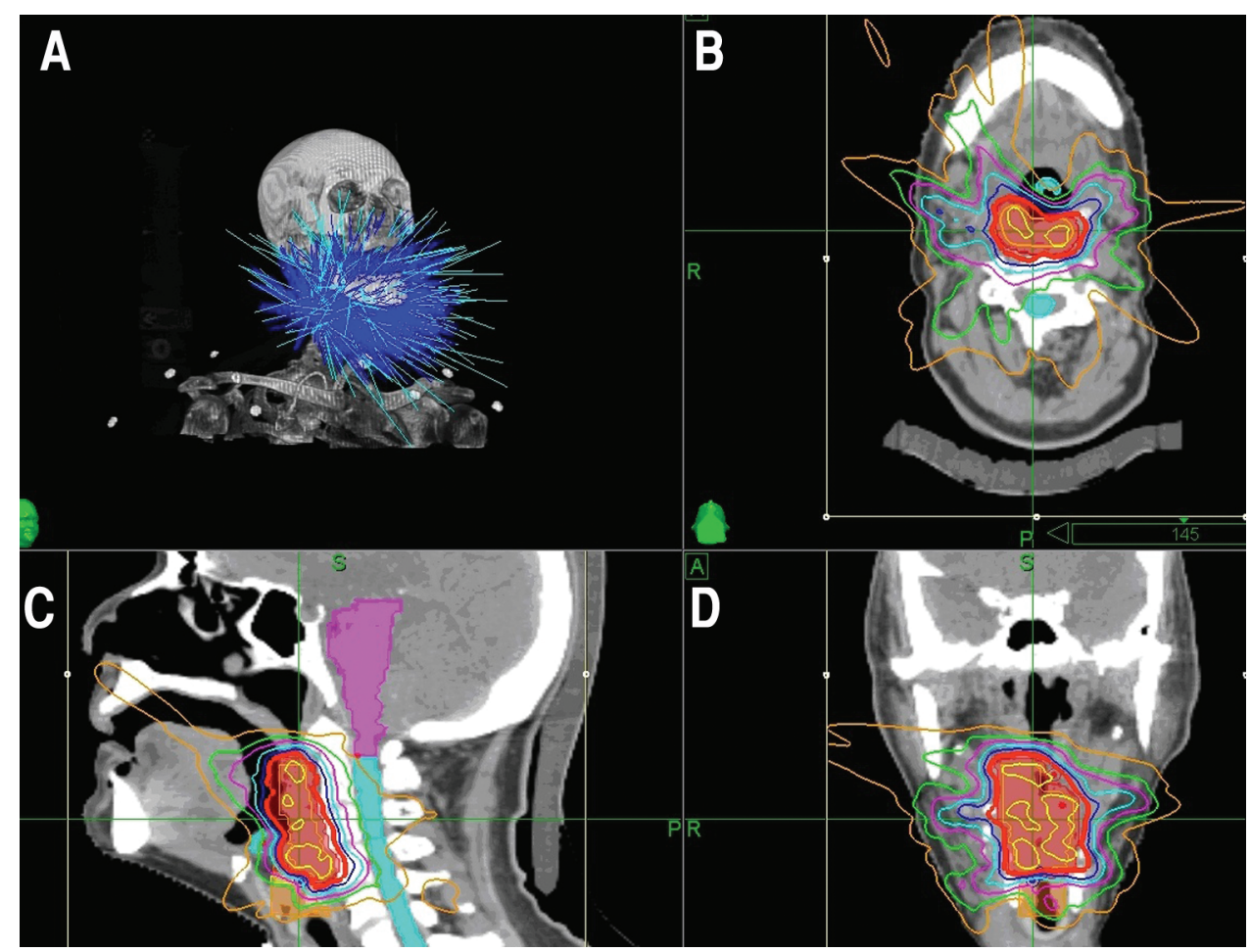

FIG. 1. Images showing an isodose plan for a patient treated with CyberKnife SBRT for a posterior hypopharyngeal wall cancer using the spine tracking method. A 3D reconstruction showing beam entries is depicted $(A)$, along with isodose distributions on the planning CT in the axial (B), sagittal (C), and coronal (D) planes.

in a location to minimize the risk of seed loss or seed migration. For this purpose we devised a protocol in which all fiducials are placed while the patient is under general anesthesia. For placement of fiducials for base of tongue or oral tongue lesions, the mouth is opened with a Jenning retractor and under continuous fluoroscopic guidance the fiducials are delivered bilaterally at least $1 \mathrm{~cm}$ lateral to the base of tongue tumor via a transoral approach. Care is taken to place both these fiducials in different planes. This placement is confirmed via $45^{\circ}$ radiographs in the operating suite using continuous fluoroscopy. In addition, a fiducial is placed in the anterior tongue. All fiducials are placed at least $3 \mathrm{~cm}$ below the surface of the tongue. An additional fiducial site that may be used includes the tonsillar pillars. Because the fiducials are inert they do not need to be removed after treatment.

The CyberKnife radiosurgery system tracks the fiducials using 2 continuous radiographic units visualizing the fiducials at $45^{\circ}$ angles, and makes corrections according to the location of the fiducial markers and adjusts its beams accordingly prior to delivering each beam, thereby reducing the error causing by tumor motion during treatment. In any 1 treatment fraction, the robotic arm may make up to 40 positional corrections during the course of beam delivery. This method is required for tumors of the base of tongue, tonsil, and other sites that can move with swallowing. Figure 2 shows a digital reconstructed radiograph of fiducial tracking in HNC.

The Xsight Spine Tracking System (Accuray), an advanced nonrigid image registration System, can automatically track lesions close to spinal structures. This system allows automated 3D target tracking technology, using orthogonal radiographs prior to delivery of each node. The images are overlaid with the digital reconstructed radiographs using deformable image registration. This system can be used for pharyngeal tumors that are adjacent to the spine. Skull tracking can be used for skull-based tumors or nasopharyngeal lesions.

At Boston Medical Center, we used fiducial tracking on all patients with oral or base of tongue cancer (procedure noted above). Gold seed fiducials were placed 5 days prior to CT simulation. One patient with a posterior hypopharyngeal wall cancer, which was adjacent to the cervical spine, underwent the Xsight spine tracking method (Fig. 3).

Gantry-Based SBRT. In the study by Siddiqui et al., ${ }^{52}$

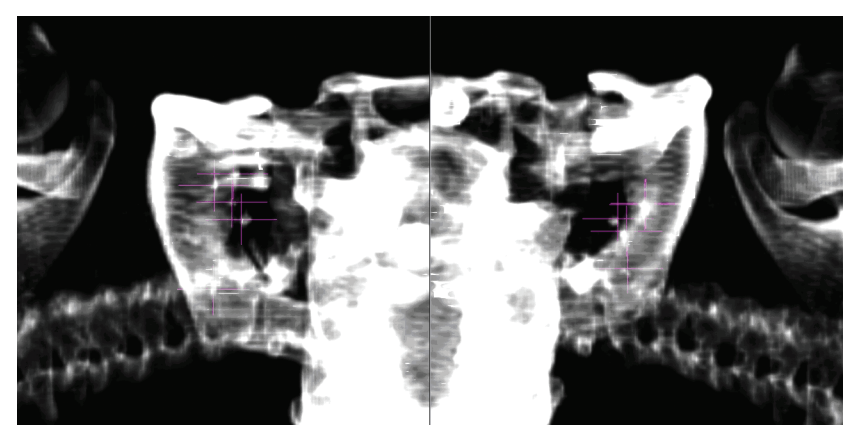

FIG. 2. Digital reconstructed radiograph of a patient with oral tongue cancer treated with CyberKnife SBRT. Pink cross hairs show the location of the fiducials. 


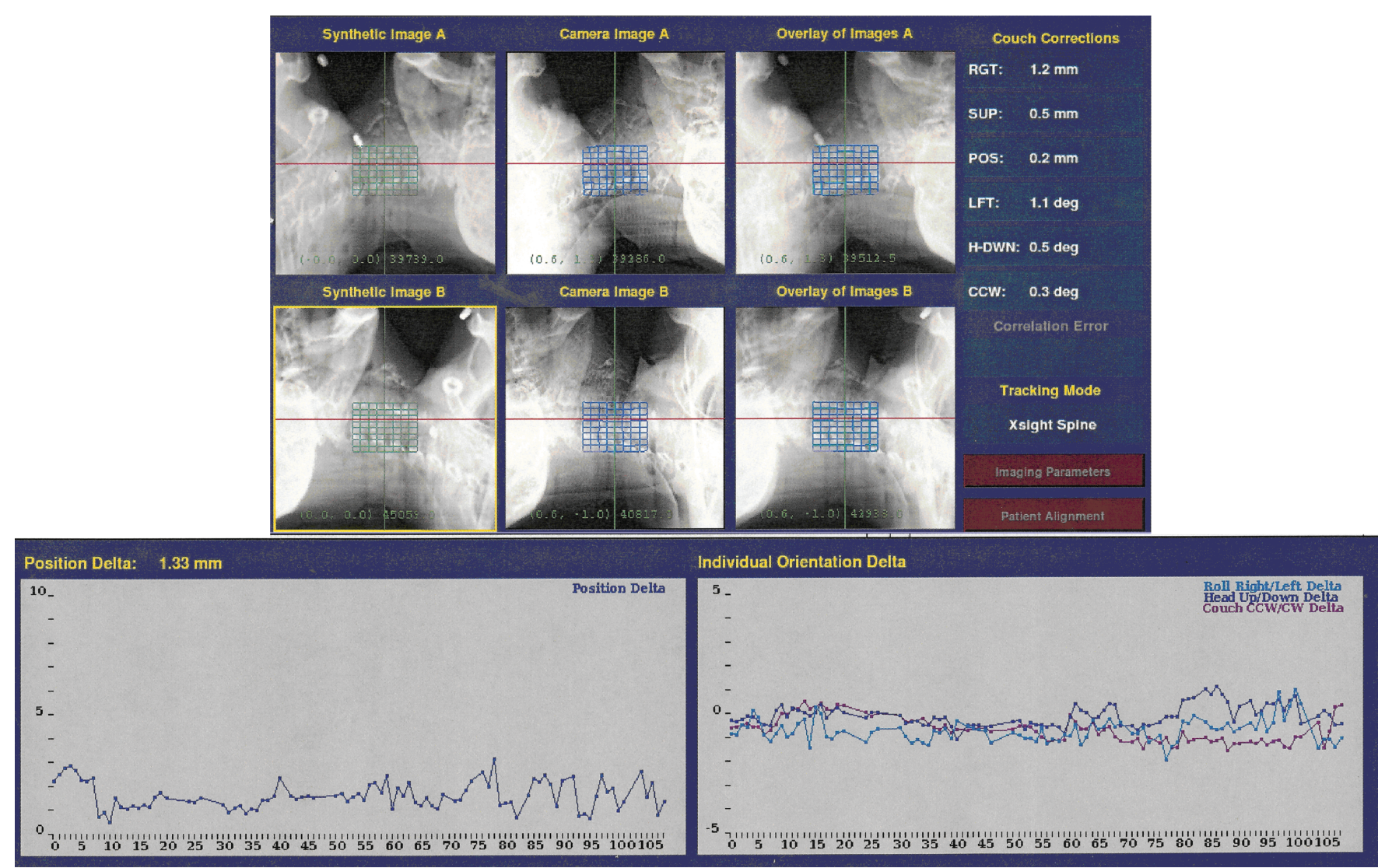

FIG. 3. Images obtained using the Xsight spine tracking method. Upper: Image showing the spine tracking method used for CyberKnife SBRT for a posterior hypopharyngeal wall cancer. Digital reconstructed radiographs from CT simulation are overlaid with real-time radiographic imaging during treatment. Lower: Image showing positional corrections made throughout treatment delivery. The $y$-axis measures the positional correction in millimeters, the $x$-axis represents the beam number. Position delta (sum of $x, y$, and $z$ coordinate shift) is the overall vector displacement from the planned imaging center to the observed imaging center for each beam. Individual Orientation Delta graphs the $x, y$, and z coordinate shifts made throughout treatment.

LINAC gantry-based SBRT was performed using BrainLAB software. Patients were immobilized in a thermoplastic mask with a custom bite block. A stereotactic head and neck localization box embedded with a Z-shaped fiducial line (using aluminum strips) was located on each side of the box to serve as a stereotactic localizer. The localization box was placed prior to each treatment and verified by kilovoltage images prior to each treatment delivery. In this study, intrafraction motion was not monitored. Linear accelerator gantry-based SBRT does have the potential to use radiographic imaging of intrafraction patient motion in head and neck treatments and has been shown to be feasible in a series of 13 patients with head and neck treatment with SBRT using the Novalis/BrainLAB system. ${ }^{46}$

\section{Evaluation of Treatment Response}

Of the 4 patients treated at Boston Medical Center, 3 patients underwent a posttherapy FDG-PET/CT scan at 8-10 weeks after completion of SBRT. According to FDG-PET/CT, 1 patient achieved a complete metabolic response, and 2 patients achieved a partial response; 1 patient has not undergone follow-up imaging. Patients were also evaluated by clinical examination and fiberoptic nasoendoscopy; 2 patients achieved a complete clinical response, and 2 patients achieved a partial response at last follow-up.

Most studies investigating SBRT in HNC used PET$\mathrm{CT}$ to evaluate radiographic response, using both $\mathrm{CT}$ response by size criteria, and PET metabolic response by standard uptake value using EORTC criteria.${ }^{64}$ Heron et al. ${ }^{34}$ showed that the PET response occurred earlier than $\mathrm{CT}$ volume changes, suggesting that PET was more sensitive in assessing treatment response. Response rates for SBRT range from 17 to $80 \%$. $^{34,51}$ The response rates after SBRT in HNC are described in Table 1 and include patients who achieved a complete and partial response. Patients who had stable disease after SBRT were not included as treatment responders. The wide variation in response may be attributed to the primary treatment site, histological type, selection of dose and fractionation, and patient selection. Stereotactic body radiotherapy used as part of the initial treatment as a boost has more favorable outcomes than SBRT used in the salvage setting for reirradiation. In particular, the high response rates using SBRT as a boost in nasopharyngeal cancer are noted in the Stanford series compared with the reirradiation series 
of all HNC sites. This finding may reflect the favorable prognosis of nasopharyngeal cancer compared with other HNC sites, and also the overall unfavorable prognosis of patients undergoing reirradiation salvage options.

\section{Quality of Life Outcomes}

Quality of life after treatment for HNC is a key component of decision-making when choosing treatment options. Surgery, or radiation therapy with or without chemotherapy, has different acute and long-term toxicity profiles with respect to functional outcome, including swallowing, voice preservation, dental complications, and visual and hearing function. Only 1 study objectively measured QOL outcomes using the revised University of Washington QOL Questionnaire. ${ }^{34}$ Quality of life in this study was noted to decline after treatment, although this study included patients who underwent reirradiation, of whom 30-50\% had significant baseline symptoms including difficulties with speech, swallowing, pain, and salivary function prior to SBRT.

At Boston Medical Center, we will be obtaining QOL measures using the EORTC Core Questionnaire QLQ-C30 and its Head and Neck Module (H\&N35). ${ }^{56}$ This module has been validated in HNC with respect to disease sites, performance status, changes in disease status over time, and cross culturally. ${ }^{8,19,47}$ Its use in CyberKnife SBRT for HNC has not been tested. We plan on comparing the concordance between physician-reported treatment toxicity via the National Cancer Institute Common Toxicity Criteria (NCI-CTC, version 3) of the US National Institutes of Health (http://ctep.cancer.gov/ reporting/ctc.html) and the EORTC Core Questionnaires for patients participating in the study.

\section{Conclusions}

The role of SBRT in HNC is an emerging treatment option for locally advanced and recurrent $\mathrm{HNC}$ as a radiosurgical boost or a form of highly focused reirradiation therapy. Phase I data shows that SBRT for HNC is a feasible treatment option with promising local control rates, although additional studies are needed to determine optimal patient selection, dose fractionation, and timing of therapy compared with other treatment strategies such as conventional radiotherapy, surgery, and chemotherapy.

\section{Disclosure}

This study was supported in part by a grant (No. 06131010) awarded to Drs. Chin and Kachnic from the Department of Defense Center of Excellence.

\section{References}

1. Adler JR Jr, Chang SD, Murphy MJ, Doty J, Geis P, Hancock SL: The Cyberknife: a frameless robotic system for radiosurgery. Stereotact Funct Neurosurg 69:124-128, 1997

2. Adler JR Jr, Murphy MJ, Chang SD, Hancock SL: Imageguided robotic radiosurgery. Neurosurgery 44:1299-1307, 1999

3. Ahn YC, Lee KC, Kim DY, Huh SJ, Yeo IH, Lim DH, et al:
Fractionated stereotactic radiation therapy for extracranial head and neck tumors. Int J Radiat Oncol Biol Phys 48:501505,2000

4. Al-Sarraf M, LeBlanc M, Giri PG, Fu KK, Cooper J, Vuong $\mathrm{T}$, et al: Chemoradiotherapy versus radiotherapy in patients with advanced nasopharyngeal cancer: phase III randomized intergroup study 0099. J Clin Oncol 16:1310-1317, 1998

5. American Cancer Society: Cancer Facts and Figures 2009. Atlanta: American Cancer Society, 2009

6. Barrett WL, Gleich L, Wilson K, Gluckman J: Organ preservation with interstitial radiation for base of tongue cancer. Am J Clin Oncol 25:485-488, 2002

7. Bernier J, Domenge C, Ozsahin M, Matuszewska K, Lefèbvre JL, Greiner RH, et al: Postoperative irradiation with or without concomitant chemotherapy for locally advanced head and neck cancer. N Engl J Med 350:1945-1952, 2004

8. Bjordal K, de Graeff A, Fayers PM, Hammerlid E, van Pottelsberghe C, Curran D, et al: A 12 country field study of the EORTC QLQ-C30 (version 3.0) and the head and neck cancer specific module (EORTC QLQ-H\&N35) in head and neck patients. Eur J Cancer 36:1796-1807, 2000

9. Bolner A, Mussari S, Fellin G, Pani G, Busana L, Caffo O, et al: The role of brachytherapy in the management of oropharyngeal carcinomas: the Trento experience. Tumori 88:137-141, 2002

10. Bonner JA, Giralt J, Harari PM, Cohen R, Jones C, Sur RK: Cetuximab prolongs survival in patients with locoregionally advanced squamous cell carcinoma of head and neck: A phase III study of high dose radiation therapy with or without cetuximab. J Clin Oncol 22 (Suppl):5507, 2004

11. Bonner JA, Harari PM, Giralt J, Azarnia N, Shin DM, Cohen $\mathrm{RB}$, et al: Radiotherapy plus cetuximab for squamous-cell carcinoma of the head and neck. N Engl J Med 354:567-578, 2006

12. Brianzoni E, Rossi G, Ancidei S, Berbellini A, Capoccetti F, Cidda C, et al: Radiotherapy planning: PET/CT scanner performances in the definition of gross tumour volume and clinical target volume. Eur J Nucl Med Mol Imaging 32:13921399,2005

13. Buatti JM, Friedman WA, Bova FJ, Mendenhall WM: Linac radiosurgery for locally recurrent nasopharyngeal carcinoma: rationale and technique. Head Neck 17:14-19, 1995

14. Calais G, Alfonsi M, Bardet E, Sire C, Germain T, Bergerot P, et al: Randomized trial of radiation therapy versus concomitant chemotherapy and radiation therapy for advanced-stage oropharynx carcinoma. J Natl Cancer Inst 91:2081-2086, 1999

15. Chao KS, Ozyigit G, Tran BN, Cengiz M, Dempsey JF, Low DA: Patterns of failure in patients receiving definitive and postoperative IMRT for head-and-neck cancer. Int J Radiat Oncol Biol Phys 55:312-321, 2003

16. Chua DT, Sham JS, Hung KN, Leung LH, Cheng PW, Kwong PW: Salvage treatment for persistent and recurrent T1-2 nasopharyngeal carcinoma by stereotactic radiosurgery. Head Neck 23:791-798, 2001

17. Chua DT, Sham JS, Kwong PW, Hung KN, Leung LH: Linear accelerator-based stereotactic radiosurgery for limited, locally persistent, and recurrent nasopharyngeal carcinoma: efficacy and complications. Int J Radiat Oncol Biol Phys 56:177-183, 2003

18. Cmelak AJ, Cox RS, Adler JR, Fee WE Jr, Goffinet DR: Radiosurgery for skull base malignancies and nasopharyngeal carcinoma. Int J Radiat Oncol Biol Phys 37:997-1003, 1997

19. Conroy T, Mercier M, Bonneterre J, Luporsi E, Lefebvre JL, Lapeyre M, et al: French version of FACT-G: validation and comparison with other cancer-specific instruments. Eur J Cancer 40:2243-2252, 2004

20. Cooper JS, Pajak TF, Forastiere AA, Jacobs J, Campbell BH, Saxman SB, et al: Postoperative concurrent radiotherapy and 
chemotherapy for high-risk squamous-cell carcinoma of the head and neck. N Engl J Med 350:1937-1944, 2004

21. Cooper JS, Pajak TF, Rubin P, Tupchong L, Brady LW, Leibel SA, et al: Second malignancies in patients who have head and neck cancer: incidence, effect on survival and implications based on the RTOG experience. Int J Radiat Oncol Biol Phys 17:449-456, 1989

22. Creak AL, Harrington K, Nutting C: Treatment of recurrent head and neck cancer: re-irradiation or chemotherapy? Clin Oncol (R Coll Radiol) 17:138-147, 2005

23. Denis F, Garaud P, Bardet E, Alfonsi M, Sire C, Germain T, et al: Final results of the 94-01 French Head and Neck Oncology and Radiotherapy Group randomized trial comparing radiotherapy alone with concomitant radiochemotherapy in advanced-stage oropharynx carcinoma. J Clin Oncol 22:6976,2004

24. Emami B, Sethi A, Petruzzelli GJ: Influence of MRI on target volume delineation and IMRT planning in nasopharyngeal carcinoma. Int J Radiat Oncol Biol Phys 57:481-488, 2003

25. Forastiere AA, Goepfert H, Maor M, Pajak TF, Weber R, Morrison $\mathrm{W}$, et al: Concurrent chemotherapy and radiotherapy for organ preservation in advanced laryngeal cancer. N Engl J Med 349:2091-2098, 2003

26. Fu KK, Pajak TF, Trotti A, Jones CU, Spencer SA, Phillips TL, et al: A Radiation Therapy Oncology Group (RTOG) phase III randomized study to compare hyperfractionation and two variants of accelerated fractionation to standard fractionation radiotherapy for head and neck squamous cell carcinomas: first report of RTOG 9003. Int J Radiat Oncol Biol Phys 48:7-16, 2000

27. Gardner M, Halimi P, Valinta D, Plantet MM, Alberini JL, Wartski M, et al: Use of single MRI and 18F-FDG PET-CT scans in both diagnosis and radiotherapy treatment planning in patients with head and neck cancer: advantage on target volume and critical organ delineation. Head Neck 31:461467, 2009

28. Gibbs IC, Le QT, Shah RD, Terris DJ, Fee WE, Goffinet DR: Long-term outcomes after external beam irradiation and brachytherapy boost for base-of-tongue cancers. Int J Radiat Oncol Biol Phys 57:489-494, 2003

29. Gleich LL, Ryzenman J, Gluckman JL, Wilson KM, Barrett WL, Redmond KP: Recurrent advanced (T3 or T4) head and neck squamous cell carcinoma: is salvage possible? Arch Otolaryngol Head Neck Surg 130:35-38, 2004

30. Hara W, Loo BW Jr, Goffinet DR, Chang SD, Adler JR, Pinto HA, et al: Excellent local control with stereotactic radiotherapy boost after external beam radiotherapy in patients with nasopharyngeal carcinoma. Int J Radiat Oncol Biol Phys 71:393-400, 2008

31. Haraf DJ, Weichselbaum RR, Vokes EE: Re-irradiation with concomitant chemotherapy of unresectable recurrent head and neck cancer: a potentially curable disease. Ann Oncol 7:913-918, 1996

32. Harrison LB, Zelefsky MJ, Sessions RB, Fass DE, Armstrong JG, Pfister DG, et al: Base-of-tongue cancer treated with external beam irradiation plus brachytherapy: oncologic and functional outcome. Radiology 184:267-270, 1992

33. Heron DE, Andrade RS, Flickinger J, Johnson J, Agarwala SS, Wu A, et al: Hybrid PET-CT simulation for radiation treatment planning in head-and-neck cancers: a brief technical report. Int J Radiat Oncol Biol Phys 60:1419-1424, 2004

34. Heron DE, Ferris RL, Karamouzis M, Andrade RS, Deeb EL, Burton S, et al: Stereotactic body radiotherapy for recurrent squamous cell carcinoma of the head and neck: results of a phase i dose-escalation trial. Int J Radiat Oncol Biol Phys [epub ahead of print], 2009

35. Heron DE, Smith RP, Andrade RS: Advances in image-guided radiation therapy - the role of PET-CT. Med Dosim 31:3-11, 2006
36. Hoffman HT, Karnell LH, Funk GF, Robinson RA, Menck HR: The National Cancer Data Base report on cancer of the head and neck. Arch Otolaryngol Head Neck Surg 124:951962,1998

37. Jemal A, Siegel R, Ward E, Murray T, Xu J, Smigal C, et al: Cancer statistics, 2006. CA Cancer J Clin 56:106-130, 2006

38. Karakoyun-Celik O, Norris CM Jr, Tishler R, Mahadevan A, Clark JR, Goldberg S, et al: Definitive radiotherapy with interstitial implant boost for squamous cell carcinoma of the tongue base. Head Neck 27:353-361, 2005

39. Kasperts N, Slotman B, Leemans CR, Langendijk JA: A review on re-irradiation for recurrent and second primary head and neck cancer. Oral Oncol 41:225-243, 2005

40. Kuo JS, Yu C, Petrovich Z, Apuzzo ML: The CyberKnife stereotactic radiosurgery system: description, installation, and an initial evaluation of use and functionality. Neurosurgery 53:1235-1239, 2003

41. Laccourreye O, Weinstein G, Brasnu D, Bassot V, Cauchois $\mathrm{R}$, Jouffre V, et al: A clinical trial of continuous cisplatinfluorouracil induction chemotherapy and supracricoid partial laryngectomy for glottic carcinoma classified as T2. Cancer 74:2781-2790, 1994

42. Le QT, Tate D, Koong A, Gibbs IC, Chang SD, Adler JR, et al: Improved local control with stereotactic radiosurgical boost in patients with nasopharyngeal carcinoma. Int J Radiat Oncol Biol Phys 56:1046-1054, 2003

43. Lefebvre JL, Chevalier D, Luboinski B, Kirkpatrick A, Collette L, Sahmoud T: Larynx preservation in pyriform sinus cancer: preliminary results of a European Organization for Research and Treatment of Cancer phase III trial. J Natl Cancer Inst 88:890-899, 1996

44. Leung TW, Tung SY, Sze WK, Sze WM, Wong VY, Wong CS, et al: Salvage radiation therapy for locally recurrent nasopharyngeal carcinoma. Int J Radiat Oncol Biol Phys 48:13311338,2000

45. Liao J, Judson B, Davidson B, Amin A, Gagnon G, Harter $\mathrm{K}$ : Cyberknife fractionated stereotactic radiosurgery for the treatment of primary and recurrent head and neck cancer. Int J Radiat Oncol Biol Phys 63 (1 Suppl):S381, 2005

46. Linthout N, Verellen D, Tournel K, Storme G: Six dimensional analysis with daily stereoscopic $\mathrm{X}$-ray imaging of intrafraction patient motion in head and neck treatments using five points fixation masks. Med Phys 33:504-513, 2006

47. Mehanna HM, Morton RP: Patients' views on the utility of quality of life questionnaires in head and neck cancer: a randomised trial. Clin Otolaryngol 31:310-316, 2006

48. Nishioka T, Shiga T, Shirato H, Tsukamoto E, Tsuchiya K, Kato T, et al: Image fusion between 18FDG-PET and MRI/ CT for radiotherapy planning of oropharyngeal and nasopharyngeal carcinomas. Int J Radiat Oncol Biol Phys 53:10511057,2002

49. Pai PC, Chuang CC, Wei KC, Tsang NM, Tseng CK, Chang $\mathrm{CN}$ : Stereotactic radiosurgery for locally recurrent nasopharyngeal carcinoma. Head Neck 24:748-753, 2002

50. Poon D, Yap SP, Wong ZW, Cheung YB, Leong SS, Wee J, et al: Concurrent chemoradiotherapy in locoregionally recurrent nasopharyngeal carcinoma. Int J Radiat Oncol Biol Phys 59:1312-1318, 2004

51. Roh KW, Jang JS, Kim MS, Sun DI, Kim BS, Jung SL, et al: Fractionated stereotactic radiotherapy as reirradiation for locally recurrent head and neck cancer. Int J Radiat Oncol Biol Phys 74:1348-1355, 2009

52. Siddiqui F, Patel M, Khan M, McLean S, Dragovic J, Jin JY, et al: Stereotactic body radiation therapy for primary, recurrent, and metastatic tumors in the head-and-neck region. Int J Radiat Oncol Biol Phys 74:1047-1053, 2009

53. Spencer S, Wheeler R, Peters G, Meredith R, Beenken S, Nabel L, et al: Phase 1 trial of combined chemotherapy and 


\section{Emerging applications of SBRT in head and neck cancer}

reirradiation for recurrent unresectable head and neck cancer. Head Neck 25:118-122, 2003

54. Spencer SA, Harris J, Wheeler RH, Machtay M, Schultz C, Spanos W, et al: RTOG 96-10: reirradiation with concurrent hydroxyurea and 5-fluorouracil in patients with squamous cell cancer of the head and neck. Int J Radiat Oncol Biol Phys 51:1299-1304, 2001

55. Spencer SA, Wheeler RH, Peters GE, Beenken SW, Meredith RF, Smith J, et al: Concomitant chemotherapy and reirradiation as management for recurrent cancer of the head and neck. Am J Clin Oncol 22:1-5, 1999

56. Sprangers MA, Cull A, Groenvold M, Bjordal K, Blazeby J, Aaronson NK : The European Organization for Research and Treatment of Cancer approach to developing questionnaire modules: an update and overview. Qual Life Res 7:291-300, 1998

57. Stevens KR Jr, Britsch A, Moss WT: High-dose reirradiation of head and neck cancer with curative intent. Int J Radiat Oncol Biol Phys 29:687-698, 1994

58. Tate DJ, Adler JR Jr, Chang SD, Marquez S, Eulau SM, Fee WE, et al: Stereotactic radiosurgical boost following radiotherapy in primary nasopharyngeal carcinoma: impact on local control. Int J Radiat Oncol Biol Phys 45:915-921, 1999

59. Voynov G, Heron DE, Burton S, Grandis J, Quinn A, Ferris R, et al: Frameless stereotactic radiosurgery for recurrent head and neck carcinoma. Technol Cancer Res Treat 5:529-535, 2006
60. Weinstein GS, El-Sawy MM, Ruiz C, Dooley P, Chalian A, El-Sayed MM, et al: Laryngeal preservation with supracricoid partial laryngectomy results in improved quality of life when compared with total laryngectomy. Laryngoscope 111:191199, 2001

61. Wong LY, Wei WI, Lam LK, Yuen AP: Salvage of recurrent head and neck squamous cell carcinoma after primary curative surgery. Head Neck 25:953-959, 2003

62. Xiao J, Xu G, Miao Y: Fractionated stereotactic radiosurgery for 50 patients with recurrent or residual nasopharyngeal carcinoma. Int J Radiat Oncol Biol Phys 51:164-170, 2001

63. Yau TK, Sze WM, Lee WM, Yeung MW, Leung KC, Hung WM, et al: Effectiveness of brachytherapy and fractionated stereotactic radiotherapy boost for persistent nasopharyngeal carcinoma. Head Neck 26:1024-1030, 2004

64. Young H, Baum R, Cremerius U, Herholz K, Hoekstra O, Lammertsma AA, et al: Measurement of clinical and subclinical tumour response using [18F]-fluorodeoxyglucose and positron emission tomography: review and 1999 EORTC recommendations. Eur J Cancer 35:1773-1782, 1999

Manuscript submitted August 17, 2009.

Accepted September 3, 2009.

Address correspondence to: Minh T. Truong, M.D., Department of Radiation Oncology, Boston Medical Center, 830 Harrison Avenue, Moakley Building LL 238, Boston, Massachusetts 02118. email: mitruong@bu.edu. 